\section{Too invasive for routine use}

\section{To the editor:}

I am concerned that the Technique paper, "A technique for retrograde intubation in mice," published in Lab Animal, Vol. 35, No. 3, could be seen by other researchers/investigators as a technique for routine intubation of mice. The authors of the article state in the discussion that this proposed technique is "a more reliable and simpler method" than direct orotracheal intubation. I strongly disagree with this statement and other statements in the article. In my opinion the key sentence of this article is the last sentence, which states that this technique "... is especially suitable for studies that involve a neck incision and in which the animal will be killed at the end of the operation." I agree with the authors on this point, as this might be the only practical use of this extremely invasive technique.

In the introduction, the authors state that direct orotracheal intubation "is difficult to learn and requires considerable practice." This sentence is misleading as I assume that the authors refer to blind intu-

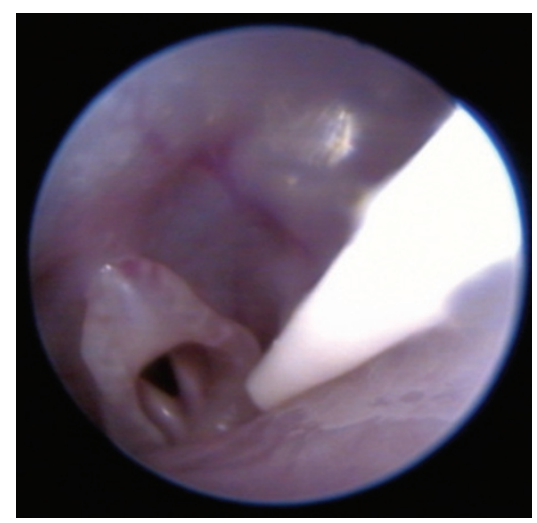

FIGURE 1 | A 14-gauge intravenous catheter is used under visual guidance to intubate a rat.

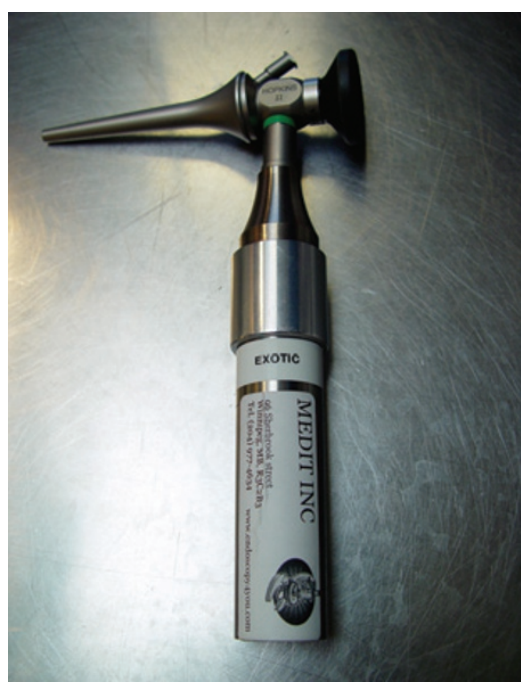

FIGURE 2 | A handheld light source combined with an otoscope is the author's preferred method of intubation of small mammals. Both pieces of equipment can be obtained from Medit Endoscopes (Winnipeg, Manitoba, Canada; http:// www.endoscopy4you.com). bation techniques without visual guidance. While the blind technique might be difficult to perform, visual intubation in the mouse can be easily performed without any special training in laboratory animal medicine or specific intubation training. An extremely useful instructional video can be seen at http://www.hallowell.com (click on "mouse intubation video" in the lower right part of the page). The kit required to perform the procedure documented in the video is available for $\sim 200$, substantially less than the quoted expense of $\$ 400-500$ for a fiberoptic guide mentioned by the authors.

The authors continue to state: "compared to direct orotracheal intubation, retrograde tracheal intubation is a more reliable and simpler method." Again, I would strongly disagree, as it seems difficult to imagine that an invasive surgery in the cervical region can be regarded as simpler than opening the mouth and inserting an endotracheal tube under visual guidance. In addition the authors report a $25 \%$ mortality rate in the animals after the surgical procedure for which retrograde intubation was used. The authors assume that all mortalities could be attributed to apparent myocardial infarction, but this is highly speculative and it would be inappropriate to draw any conclusions from the mortality data without controlled studies truly assessing the effect of this invasive intubation technique on morbidity and mortality.

The authors quote an abstract published by Deyo and $\mathrm{Wei}^{1}$, which mentions fatal complications from the direct intubation of mice. However, I was unable to locate the original article and it is difficult to judge the cause of the reported death of the three animals mentioned without having access to the original journal article.

An additional concern regarding the retrograde technique is the potential for causing iatrogenic trauma to the arytenoid cartilages. Figure 1 illustrates the anatomical position of the arytenoids relative to the endotracheal tube during the intubation procedure. It is very easy to damage the cartilage by repeatedly hitting the dorsal aspect of the cartilage with the endotracheal tube, even with a guide wire passed between the two cartilages guiding the tube in the right general direction. This is especially frequent if the endotracheal tube is slightly larger than optimal and if no local anesthesia has been applied directly to the cartilage. The slightest contact of the endotracheal tube to the cartilage will cause the arytenoids to close as part of the swallowing reflex. Damage to the cartilage can be completely eliminated by using the visual intubation technique.

In short, while I applaud the authors for their description of a refined intubation technique in mice, in my opinion, the proposed procedure should not be used for intubation when cervical surgery is not necessary. My preferred technique of intubation of small rodents is under direct visual guidance using a handheld light source and an otoscope (Fig. 2).

\section{Jörg Mayer, Dr. Med Vet., MSc}

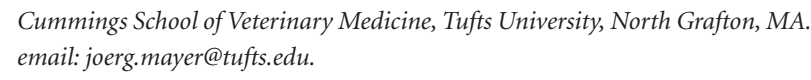

1. Deyo, D.J. \& Wei, J. Anesth. Analg. 88, S176 (1999). 